\title{
Could functional oils solve the antibiotic resistance problem?
}

\begin{abstract}
Antibiotics have traditionally
been used to control disease in farmed animals, such as broile chickens and cattle. However with the rise in antibiotic resistance, many countries have banned the use of antibiotics his colleagues at Oligo Basics, his colleagues at Oligo Basics, investigating novel alternatives to antibiotics. They are focusing their efforts on plant-derived functional oils. A mix of castor oil and cashew nut-shell liquid has been shown to effectively in broiler chickens.
\end{abstract}
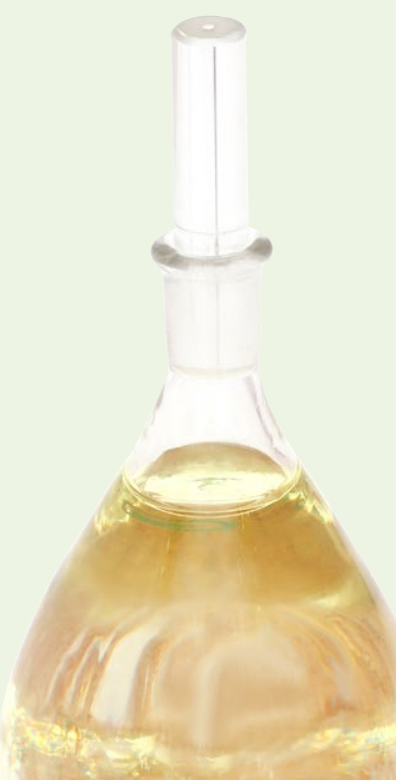

ince the discovery of penicillin
in 1928 , antibiotics have revolutionised the treatment of disease. Innumerable lives have bee saved by the ability of antibiotics to eliminate bacterial infections. Now, though, antibiotic resistance in bacteria been well-reported in humans; infections such as MRSA (Methicillin-resistant Staphylococcus aureus) are often called "superbugs" by the media, due to their resistance to common antibiotics. However, antibiotic resistance is also on the increase in farmed animals.

The conditions in which some farmed animals are raised lead to a heightened risk of bacterial infection. This is particularly true of animals such as broiler chickens, where many birds are kept in a small space. Traditionally, this problem was tackled with vaccines an antibiotics. Antibiotics have effectively been used as growth promoters, as they increase productivity as we

In response to the rise of antibiotic resistance, many countries have imposed a full or partial ban on the use of antibiotics as growth promoters in farmed animals. Consumer perception of antibiotic use in food animals is also generally negative. alternatives to antibiotics is growing

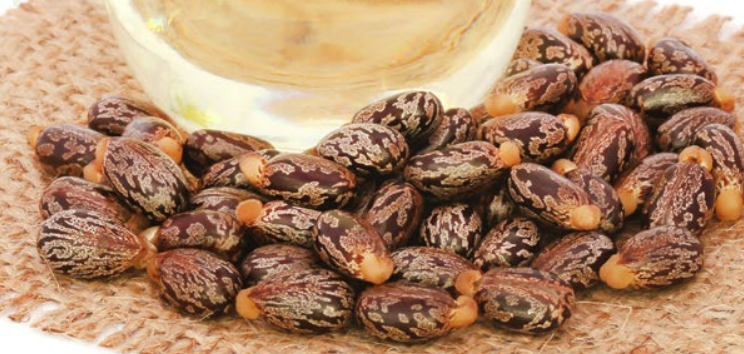

INTRODUCING: FUNCTIONAL OILS Dr Joan Torrent and his colleagues at Oligo Basics are at the forefront of the search for effective alternatives to antibiotics for use in farmed animals. They are focusing their efforts on the vegetable kingdom. For thousands of traditional remedies for illness in both humans and animals. These substances of plant origin are known as phytogenic compounds.

The researchers at Oligo Basics are taking a scientific approach to their investigation of phytogenics, to identify the active molecules and mode of action of these substances. The also looking for synergies substances are combined to create more beneficial effect.

Dr Torrent and his colleagues are specifically investigating a group of oils. These are oils that can be function to have functions beyond simply their energy value Research has suggested that some functional oils have both antibiotic and antimicrobial properties and can effectively tackle bacterial infections. Functional oils also seem to have additional, unexpected positive qualities, including antioxidant and anti-inflammatory effects. For example, castor oil has been shown to act

\section{DO FUNCTIONAL}

\section{OILS REALLY WORK?}

Dr Torrent and Oligo Basics have already been able to publish a

substantial amount of research showing positive effects. Much of the rese

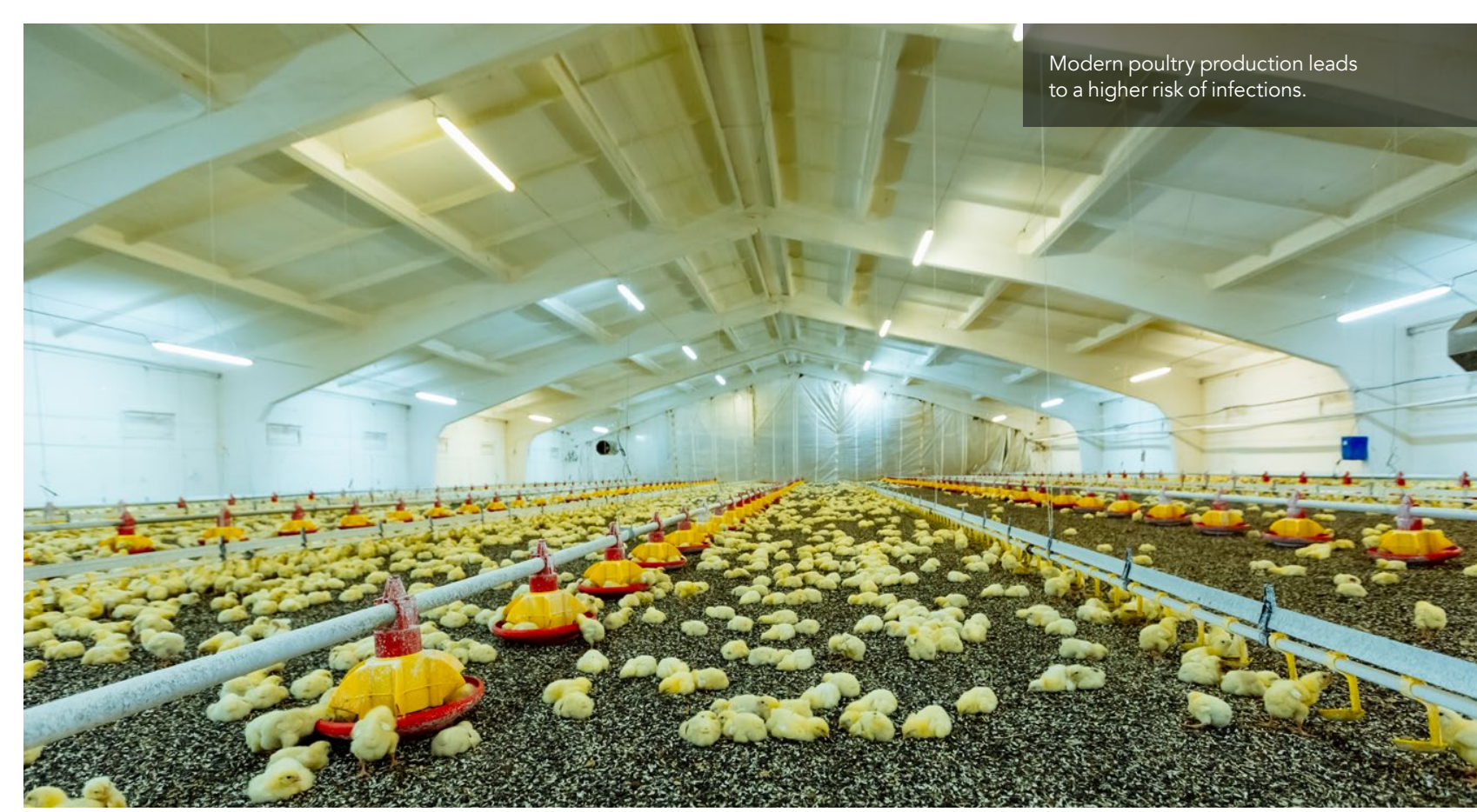

has focused on using functional oils in broiler chickens, as these are relatively cheap, easy to keep and grow quickly compared to other farmed animals.

In one study, researchers investigated the effects of functional oils on coccidiosis, a common infection in broiler chickens. Coccidiosis is caused by coccidia, a single-celled protozoan parasite, rather than a bacterium. The parasite lives in the intestinal walls of the chicken. Signs of coccidiosis in chickens include diarrhoea, weakness, a pale comb or skin and a reduced growth rate.

The researchers used a mix of two function lif (CNSL). This cashew blend is an Oligo Basics product called Essential. Castor oil, when given orally, is well-known for having laxative effects, which limit its usefulness. However, when combined with CNSL, castor oil becomes biologically active at lower doses, which avoids the laxative problem. The active molecule in castor oil is called ricinoleic acid, which has been shown to have both antimicrobial and anti-nflammatory properties. This makes it ideal for treating intestinal diseases such as coccidiosis. CNSL has also been found to have a number of useful properties,

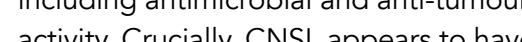

synergistic effects when combined with other antimicrobials like castor oil.

The researchers carried out their study using young male broiler weight gain became even after this time).

Some functional oils have both antibiotic and antimicrobial properties and can effectively tackle bacterial infections. into five groups: one (the control) with The control group also had a higher coccidiosis infection and no functional oil mortality rate. Chickens that received the cupplosis in to have

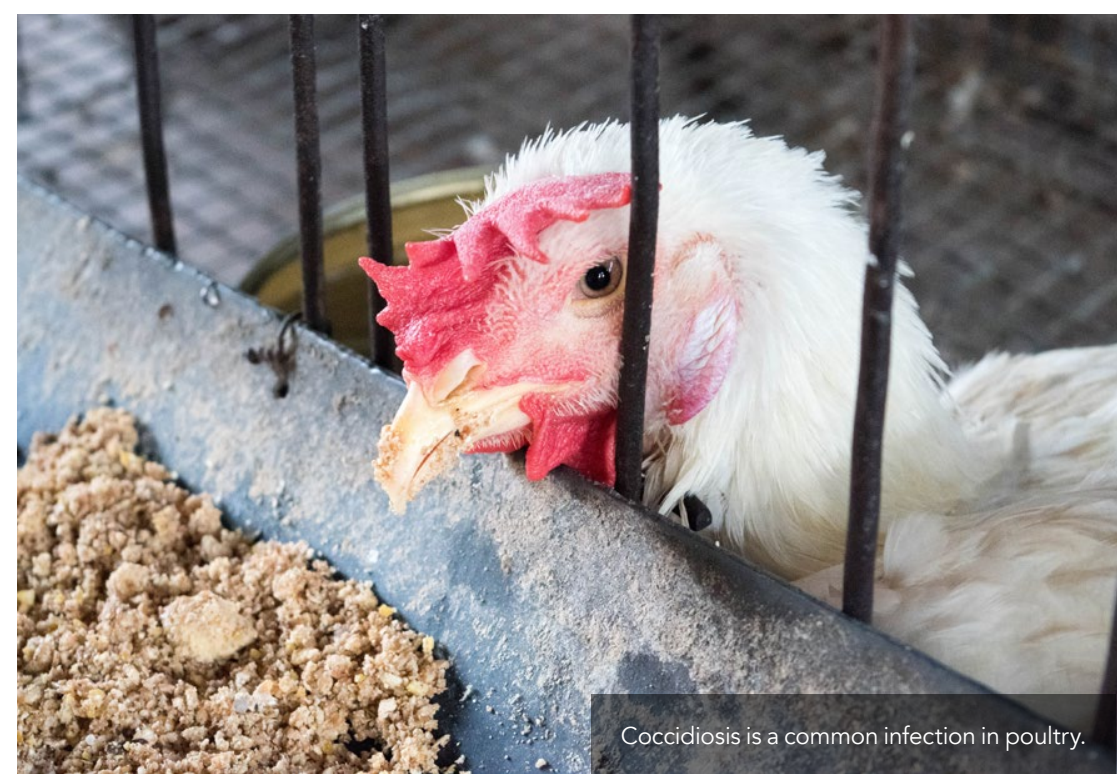




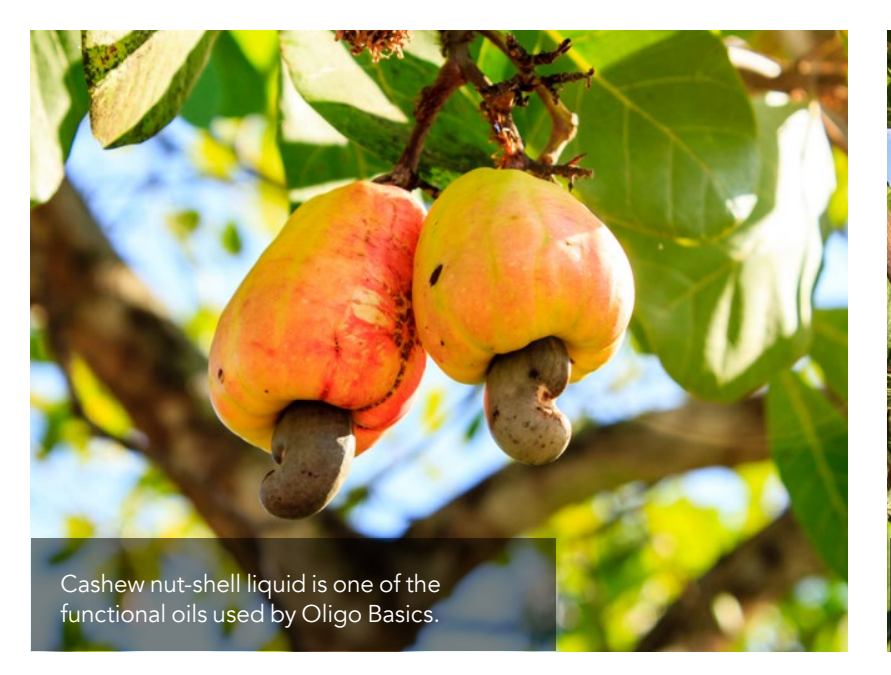

In another study, researchers found that functional oils were able to reduce the effects of heat stress in broiler chickens,
Broiler chickens can particularly suffer Broiler chickens can particularly suffer and the conditions they are kept in In this case, the researchers thopth. was likely to be the antioxidant and ant

Initially, at seven days after infection with coccidiosis, chicks treated with monensin appeared to be doing beter than those given the oil. Yet, by 14 days post-infection, the birds given were showing a better feed conversion rate than those treated with monensin.

Crucially, CNSL appears to have synergistic effects when combined with other antimicrobials.

inflammatory properties of the functional oils that had the positive effect.

A third study compared Essential, the castor oil-CNSL mix, with the antibio monensin. Monensin is commonly added to chicken feed to control coccidiosis. Young male broiler chicks were divided into groups, each of

By the end of the study, there was no difference in weight, and weight gain between chicks treated with Essenti and monensin. However, there was an even more Howeresting outcons. FUNCTIONAL OILS TARGET SPECIFIC MICROBES Researchers found that, while function

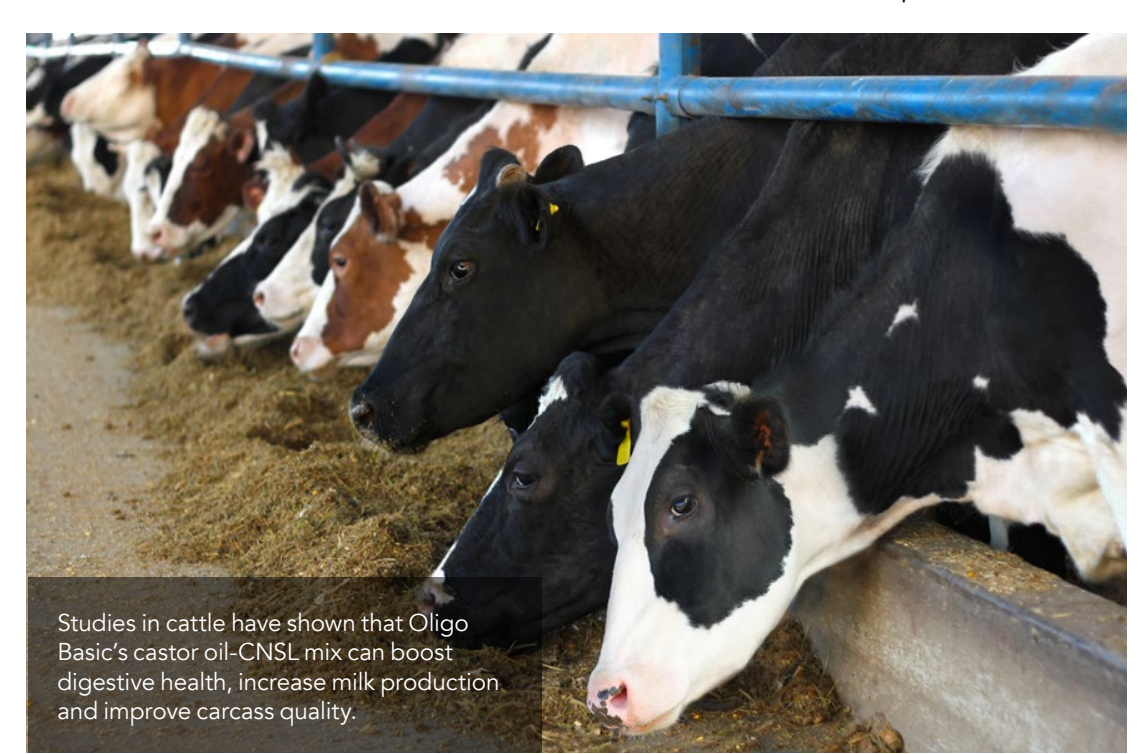

similar results in terms of weight gain, the oils are actually more specific activity activity. This is an important finding, oils will not damage the digestive mis wil namage the digestive of bacteria and other microbes found in the intestines - in the way that some antibiotics can. Instead, functional oil. treatment allows the diversity of the digestive microbiome to be maintained. This allows the animal to retain a greater ability to fight digestive diseases with its natural defences.

The research carried out by Oligo Basics to date clearly suggests that phytogenic substances, in particular functional oils, have huge potential for fighting disease in farmed animals. In addition to the work with broiler chickens, studies in cattle have show boost digestive helth, increan production and improve carcass quality. This mix of oils has been proven to be highly synergistic, with the addition of CNSL both eliminating the laxative problem and actually increasing the antimicrobial potential of castor oil.

With researchers struggling to tackle the ever-increasing problem of antibiotic resistance, new, less traditional avenues of research may become more commo So far, the research spearheaded by Dr Torrent and his colleagues has clearly shown that functional oils are not only able to act as a substitute for antibiotics, but also offer other benefits that can both improve the welfare of farmed animals, by reducing disease,

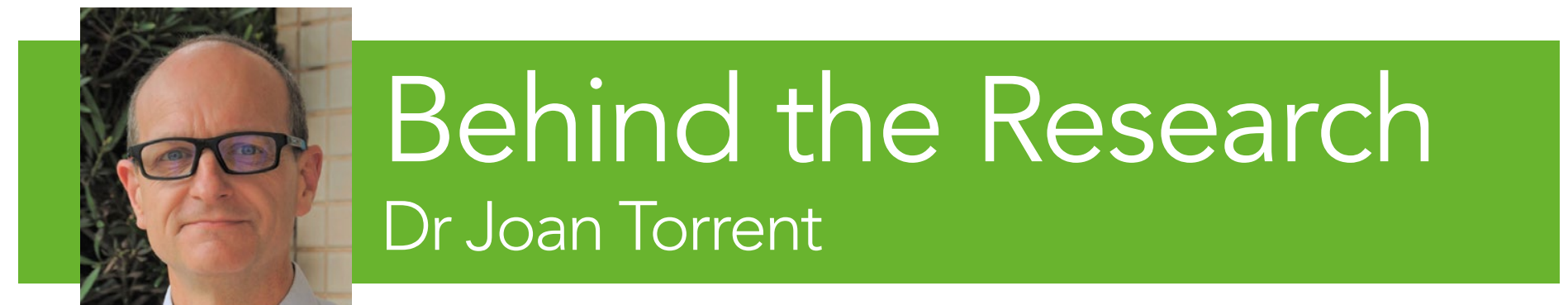

E: jtorrent@oligobasics.com T: +19524516968 W: www.oligobasics.com
Research Objectives

Oligo Basics investigates and promotes the use of functional oils as a replacement for routine antibiotic use in animals.

\section{Detail}

Rua Sérgio Gasparetto, 503

Parque Industria Cascavel, Paraná, B

Bio

Dr Joan Torrent is Global Technical Director and co-founder of Oligo Basics. Prior to that he received his PhD in Anim Nutrition from Colorado State University in 1994 and worked in research and development of different private companies in Europe and Latin America.

Funding
Oligo Basics

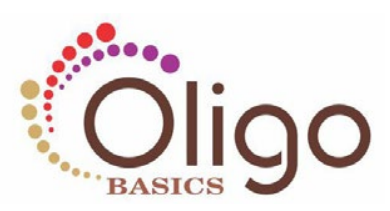

\section{References}

Bess, F., et al. 2012. The effects of functional oils on broiler diets of varying energy levels. J. Appl. Pout. Res. 21:567-578.

Moraes, P. O. et al. 2019 Comparison between a commercial blend of functional oils and monensin on the performance and microbiota
broilers. Poultry Science 0:1-9.

Murakami, A. E. , Eyng, C \& Torrent, J. 2014. Effects of functional oils on coccidiosis and apparent metabolizable energy in broiler ch
Sci. 27(7): $981-989$.

\section{Personal Response}

Other than castor oil and CNSL, are any other functional oils showing potential as antibiotic substitutes? II Other examples of functional oils are: 1) medium chain fatty acids (caproic, caprylic, capric, and lauric acids) that the primary energy source of intestinal epithelial cells, has immunomodulatory effects. strong antimicrobial activity; 2) butyrate that besides being

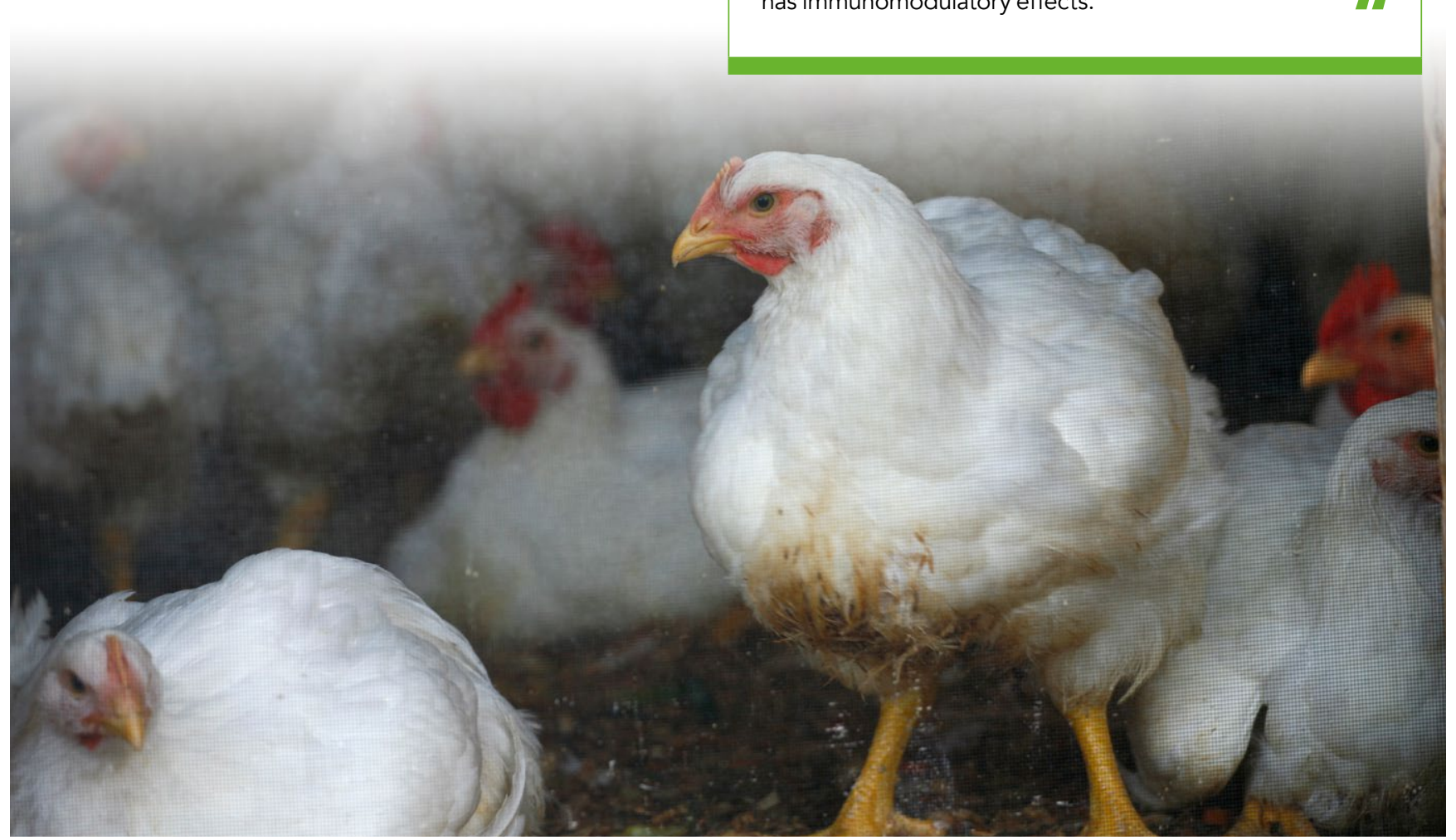

